\title{
Widespread ocular use of topical chloramphenicol: is there justifiable concern regarding idiosyncratic aplastic anaemia?
}

\author{
Charles N J McGhee, Constantine N Anastas
}

Seldom has an article, which merely regurgitates previously widely known information, produced such a wide response in the ophthalmic and medical community as the recent editorial in the $B M \mathcal{F}$ by Doona and Walsh. ${ }^{1}$ However, mention of 'litigation' or suggestion of negligent practice focuses the medical mind marvellously. While accident and emergency departments around the UK suspend the use of topical chloramphenicol, general practitioners question its safety in children, and ophthalmologists, ${ }^{23}$ microbiologists, ${ }^{4}$ and haematologists ${ }^{5}$ rush to its defence, a realistic reassessment of the role of topical chloramphenicol in eye care is desirable.

Chloramphenicol is an antibiotic produced by Streptomyces venezuelae originally isolated in 1947 and introduced into clinical practice in $1948 .^{6-8}$ It comprises a nitrobenzene ring linked to propanol with an amide group binding to a derivative of dichloroacetic acid. ${ }^{7}$ Only after considerable widespread systemic use was the rare but serious side effect of idiosyncratic chloramphenicol induced aplastic anaemia identified ${ }^{8}$ with the first landmark fatal case documented in $1950 .{ }^{6}$ Currently, systemic chloramphenicol has very limited specific indications (severe enteric fever, bacterial meningitis with unknown organisms in children under 5 years, invasive Haemophilus influenzae infections, and severe rickettsial infections where tetracyclines are contraindicated). ${ }^{7}$ However, the ocular use of topical chloramphenicol remains very popular in ophthalmology and general practice, being prescribed in $55 \%$ of 'red eyes'.9 Indeed, some would suggest this is the 'gold standard' ocular antibiotic against which other antibiotics should be compared. ${ }^{2}$

Chloramphenicol is a bacteriostatic antibiotic which inhibits bacterial synthetic mechanisms by reversibly binding to the $50 \mathrm{~S}$ subunit of the $70 \mathrm{~S}$ ribosome thus preventing successful attachment of complete transfer RNA to the ribosome and consequently disrupting peptide bound formation and protein synthesis. ${ }^{71011}$ The bacteriostatic concentration for chloramphenicol is $1 \mu \mathrm{g} / \mathrm{ml} .^{12}$ Chloramphenicol undergoes primarily hepatic metabolism ${ }^{101113}$ with $93 \%$ renal excretion of metabolites in 24 hours in humans. ${ }^{13}$ The main metabolite implicated in the marrow toxicological action is nitroso-chloramphenicol (NOCAP), but dehydro-chloramphenicol (DH-CAP) and, to a lesser extent, dehydro-chloramphenicol base (NPAP) both produced by enterobacteria of the colon, also have proved cytotoxic and genotoxic actions in vitro while chloramphenicol itself and other intermediates have much less toxic action. ${ }^{13}$ Yunis demonstrated gastrointestinal flora produce chloramphenicol metabolites that may precipitate marrow aplasia and it is recognised that parenteral administration may be associated with lower risk of bone marrow toxicity. ${ }^{7}$

Topical chloramphenicol remains popular partly because of its wide spectrum of action, being highly effective against most Gram positive and Gram negative pathogens ${ }^{7}$ as well as most anaerobes (including all obligate anaerobes) ${ }^{10}$ together with mycoplasma, rickettsia, and spirochaetes. ${ }^{11}$ Enterobacteriaceae are variably resistant while pseudomonas and mycobacteria are usually resistant. ${ }^{7}$ It has a high reported efficacy of $91 \%$ to $93 \%$ in ocular infections ${ }^{1415}$ and is active against up to $94 \%$ ocular pathogens. ${ }^{9}$ Chloramphenicol also has a high differential solubility giving high intraocular penetration (10000 times more fat soluble than penicillin), ${ }^{11}$ making it superior to most other antibiotics for prophylaxis in ocular surgery, ${ }^{1216}$ with penetration of ointment noted to be superior to eyedrop preparations (single application of $1 \%$ ointment as effective as 13 applications of eyedrop preparation every 15 minutes). ${ }^{12}$ In contrast, Doona and Walsh in their recent publication ${ }^{1}$ actually suggest framycetin and fusidic acid as equally effective options in the treatment of most superficial eye infections. There are no recent published reports on framycetin sensitivities other than company information dating back to the $1960 \mathrm{~s}$, suggesting the limited bacterial sensitivities to this antibiotic are Gram negative organisms and Staph aureus, while Pseudomonas and some strains of $E$ coli, Shigella, and Salmonella are resistant. Cross resistance with neomycin exists and resistance is reported to be fairly common. Adverse effects of topical framycetin include hypersensitivity, irritation, burning, stinging, dermatitis, and a theoretical risk of ototoxicity if used in large doses in children or patients with renal or hepatic failure. ${ }^{17}$ Although fusidic acid has been reported to be as effective as chloramphenicol in some studies in the treatment of conjunctivitis, ${ }^{15}$ its range of bacterial sensitivity is mainly Gram positive organisms: Staph aureus; Streptococcus; Neisseria; Haemophilus; Moraxella; Corynebacterium, and Bacteroides; while most Gram negative organisms are resistant. Adverse effects of topical fusidic acid include hypersensitivity and stinging. ${ }^{17}$ However, ciprofloxacin, a fluoroquinolone with high ocular penetration, ${ }^{18}$ good ophthalmic tolerance and a broad spectrum of antibacterial activity may represent a more suitable alternative to chloramphenicol in resistant ocular infections. ${ }^{19}$ None the less, while the exact mechanisms by which ocular bacteria develop antibiotic resistance remain to be elucidated, it would not seem unreasonable to reserve such newer broad spectrum antibiotics for the treatment of microbial keratitis or ocular infections resistant to first line antibiotics.

\section{Adverse reactions}

Topical chloramphenicol is generally very well tolerated with uncommon local adverse effects of hypersensitivity and transient burning/stinging. ${ }^{12} 14$ Systemic chloramphenicol, in addition to lesser adverse reactions such as gastrointestinal disturbance and rash, is also associated 
with the much more serious and potentially lethal effects of bone marrow toxicity. This can take the form of dose related marrow suppression involving one or more cell lines which may or may not be reversible or the much more rare idiosyncratic aplastic anaemia. Aplastic anaemia from any cause has an overall mortality of $50 \% .^{820}$ Idiopathic aplastic anaemia has a reported incidence of 1 in $524000^{21}$ and in the most comprehensive study on the matter ever published, the incidence of chloramphenicol induced aplastic anaemia was reported as 1 in 24500 to 40800 with the incidence of chloramphenicol induced aplastic anaemia (from systemic use) being 13 times that of the idiopathic form (22 500 deaths of all causes were screened over an 18 month period in a population where an estimated 330000 people per annum were exposed to systemic chloramphenicol). ${ }^{21}$ It was noted in this study that marrow toxicity may not manifest until the second or third course of treatment. The usual adult systemic dose of chloramphenicol is 3 to $5 \mathrm{~g}$ per day in divided 4 hourly doses $^{11}$ while one drop $(0.05 \mathrm{ml})$ of guttae chloramphenicol $0.5 \%$ contains $0.25 \mathrm{mg}$ of chloramphenicol ${ }^{22}$ meaning that a typical course of topical treatment would provide a total of less than $10 \mathrm{mg}$ delivered to the eye, with only a fraction of this amount being absorbed systemically.

Many authors quote two pathogenetically different toxic effects on bone marrow, originally proposed by Yunis and co-workers and recently cited by Doona and Walsh. ${ }^{123}$ It is quite possible, however, that these two groups are bimodal ends of a spectrum. The more common, reversible, dose dependent toxicity (primarily affecting erythroid cells) is characterised by mild anaemia, thrombocytopenia, and neutropenia with mild marrow hypocellularity and is thought to be the result of mitochondrial injury. ${ }^{2324}$ The second presentation of marrow toxicity is the very rare, idiosyncratic, irreversible aplastic anaemia comprising a progressive pancytopenia and marrow hypocellularity which is apparently not related to dose or duration. ${ }^{23} 24$ There is evidence to suggest that chloramphenicol induced bone marrow aplasia occurs as the result of a genetically determined biochemical predisposition to toxicity involving the DNA synthetic pathway in replicating haematopoietic stem cells. ${ }^{23} 25$ It has been postulated that a nitroreduction derivative of chloramphenicol, or another metabolite produced by predisposed patients, may actually be produced in the marrow rather than the liver, thus making the marrow both the site of metabolic conversion and the target of injury. ${ }^{26} \mathrm{~A}$ genetic predisposition is supported by reports of systemic chloramphenicol drug induced aplastic anaemia in identical twins. ${ }^{25}$ If the susceptibility to chloramphenicol requires a homozygous state relative to a single recessive gene, then one could reasonably expect a significant incidence in non-twin siblings, which has not been demonstrated, and it is therefore suggested that an unusual combination of genetic factors must be present in a homozygous form before this abnormal susceptibility becomes manifest. ${ }^{825}$ However, with regard to the two broad groups of marrow toxicity, it is notable that a number of cases fall between or overlap these two groups and the possibility of a single pathogenic mechanism cannot be excluded. Indeed in one identical twin report, exposure to chloramphenicol resulted in aplastic anaemia in one twin and a readily reversible isolated cytopenia in the other. ${ }^{8}$

The authors are aware of 23 reported cases of blood dyscrasias (including 12 deaths) possibly related to the use of topical chloramphenicol. ${ }^{26}$ The first report was in 1965 occurring in an otherwise healthy male with a family history of chloramphenicol (systemic) induced aplastic anaemia who had used topical chloramphenicol inappropriately in an unsupervised continuous fashion for 23 months. Thirty months after the onset, red and white cell variables had normalised while a mild thrombocytopenia persisted. ${ }^{27}$ (No death occurred in this case as erroneously quoted by Doona and Walsh. ${ }^{1}$ ) The second case reported in 1975 was another non-fatal case of aplastic anaemia ${ }^{28}$ which other authors have thought to be coincidental. ${ }^{22}$ The third case (and first fatality) was actually only reported in $1980 .{ }^{24}$ The fourth case report (and second fatality) ${ }^{23}$ with the three previous reports are serially referenced by Doona and Walsh with a preceding statement, Numerous subsequent articles directly implicated chloramphenicol eye drops in causing bone marrow aplasia'. ${ }^{1}$ (In two of the four cases, other drugs could possibly have been implicated.) On close review of the published reports, it is felt by the authors that this statement would appear to be too conclusive. In the most recent and extensive report ${ }^{26}$ reviewing 23 cases (seven published), the authors acknowledge that although all reporting ophthalmologists and haematologists believed there was a possible cause and effect relation, it would be doubtful if chloramphenicol was causally related in all cases because of the background incidence of idiopathic aplastic anaemia in the general population.

\section{Justifiable concern?}

To prove causation between drug therapy and blood dyscrasias is difficult ${ }^{20}$ and some authors feel that to prove causation between topical chloramphenicol and induced aplastic anaemia would be impossible. ${ }^{23} \mathrm{~A}$ definitive cause and effect relation might be impossible to determine because: (1) toxicity is unpredictable; (2) mortality is high and marrow studies for cell toxicity are not possible except in recovery; and (3) recovery may occur through evolution of a chloramphenicol resistant clone of haematopoietic cells, in which case studies would be misleading on these patients.

Delay between exposure to chloramphenicol and occurrence of drug induced aplastic anaemia clouds the relation. ${ }^{8}$ Of those blood dyscrasias induced by systemic chloramphenicol, only $22 \%$ of cases manifest the dyscrasia while on the drug, with a median delay of 38 days to onset of dyscrasia from discontinuation of chloramphenicol and in $10 \%$ of cases the dyscrasia occurs more than 130 days after discontinuation. ${ }^{8}$ Further confounding variables might be failure to identify earlier exposure to chloramphenicol (especially because of this delayed onset) biasing towards underestimation of associated toxicity, although the latter is almost certainly countered by the high awareness of physicians of chloramphenicol induced aplastic anaemia, possibly leading to over incrimination of chloramphenicol, as opposed to other candidate drugs known to cause idiosyncratic aplastic anaemia such as sulpha based drugs and phenothiazines. ${ }^{711}$

In respect of risk associated with topical therapy, the authors feel that unfounded published statements such as 'Actually there are no data to support the belief that the incidence is any less than after systemic therapy'29 can only serve to confuse and cause panic in the prescribing physician. Considering the very widespread level of prescription of topical chloramphenicol, one would expect more than 23 published 'possibles' in the literature to date if the risk were to equal that of systemic therapy, ${ }^{21}$ which obviously represents an absolute theoretical maximum for topical therapy. However, it is acknowledged that spontaneous reporting may not necessarily equate with absolute incidence. Doona and Walsh ${ }^{1}$ postulate that the risk of topical chloramphenicol may be similar to that of systemic therapy on the basis that 'topical administration can achieve systemic effects by absorption through the conjunctival membrane or through drainage down the lacrimal duct' citing a letter ${ }^{30}$ which actually discusses the quite distinctly different scenario of subconjunctival administration of 
chloramphenicol in rabbits. While it is acknowledged that minuscule amounts of drug can theoretically precipitate idiosyncratic reactions, Trope et $a^{22}$ performed a study in children to determine systemic absorption of topical chloramphenicol concluding that significant absorption directly from the eye into the circulation or via the nasolacrimal system and gastrointestinal tract did not occur, or that the urinary drug levels were lower than that detectable by the analytical technique (working assay limit $1 \mu \mathrm{g} / \mathrm{ml}$ ). The authors, however, did go on to express concern regarding systemic absorption associated with 'excessively prolonged usage' which was not specifically addressed by their sensitive study. It should be noted that the minimum reported total topical dose of chloramphenicol proposed to be associated with marrow toxicity is $30 \mathrm{mg}$ and the minimum associated duration of exposure is 18 days. ${ }^{26}$

\section{Conclusions}

A theoretical but as yet not conclusively proved risk of chloramphenicol induced idiosyncratic aplastic anaemia exists with topical ophthalmic therapy, with the absolute, but highly improbable, maximum risk of death (equalling that of systemic therapy) being 1 in 50000 to $90000 .^{8221}$ To put this in realistic perspective, one must note that the comparable risk of fatal anaphylaxis resulting from penicillin therapy, from any route, is similar at 1 in $100000 . .^{31}$ Indeed, it has been noted recently that with more than 200 million ocular chloramphenicol products dispensed in the $\mathrm{UK}$ in the past 10 years, only 11 reports (all non-fatal) of suspected topical chloramphenicol induced blood dyscrasia have been reported to the Committee on the Safety of Medicines since $1966 .{ }^{4}$ One also has to consider that inadvertent exposure to minute quantities of chloramphenicol ( $\mathrm{ng} / \mathrm{ml}$ ) may occur through consumption of livestock that have been treated with chloramphenicol. 133233

Broad statements condemning topical chloramphenicol need to be tempered with its proved safety, tolerance, cost, and efficacy while acknowledging an extremely remote risk of the very serious adverse effect of drug induced aplastic anaemia. Risk-benefit assessment is the duty of all prescribing physicians and a decision to prescribe or not prescribe must be made on the basis of personal judgment and an awareness of the statistics in perspective. The only known factor to be associated with vulnerability in the case of topical chloramphenicol is family history. ${ }^{23}$ There is no evidence to date that suggests children are any more susceptible than adults. ${ }^{8}$

Corneal Diseases and Excimer Laser Unit,

CHARLES N J MCGHEE

Sunderland Eye Infirmary, Sunderland SR2 9HP and

University of Sunderland, School of Health Sciences,

Sunderland SR2 7EE

CONSTANTINE N ANASTAS

Corneal Diseases and Excimer Laser Unit,

Sunderland Eye Infirmary, Sunderland SR2 9HP
1 Doona $\mathrm{M}$, Walsh JB. Use of chloramphenicol as topical eye medication: time to cry halt? [Editorial]. BMF 1995; 310: 1217-8.

2 Kirkness CM, Seal DV, Hay J. Topical chloramphenicol: use or abuse? Eye 1995; 9 (4): vii-viii.

3 Buckley RJK, Kirkness CM, Kanski JJ, Ridgway AEA, Tullo AB, Watson PG. Is it time to stop using chloramphenicol on the eye? Safe in patients PG. Is it time to stop using chloramphenicol on the eye? Safe in pation
with no history of blood dyscrasias [Letter]. BMf 1995; 311: 450 .

$4 \mathrm{Hall} \mathrm{AV}$, Das SS, Tabaqchali S. Is it time to stop using chloramphenicol on the eye? Risk is low in short courses [Letter]. BMf 1995; 311: 450-1.

5 Gordon-Smith EC, Marsh JCW, Geary CG. Is it time to stop using chloramphenicol on the eye? Prospective study of aplastic anaemia should give definitive answer [Letter]. BMF 1995; 311: 451 .

6 Rich ML, Ritterhoff RJ, Hoffman RJ. A fatal case of aplastic anaemia following chloramphenicol (chloromycetin) therapy. Ann Intern Med 1950; 33: 1459-67.

7 Flegg P, Cheong I, Welsby PD. Chloramphenicol: are concerns about aplastic anaemia justified? Drug Safety 1992; 7: 167-9.

8 Best WR. Chloramphenicol-associated blood dyscrasias. FAMA 1967; 201: 181-8.

9 Buckley SA. Survey of patients taking topical medication at their first presentation to eye casualty. BMF 1990; 300: 1497-8.

10 Smilack JD, Wilson WR, Cockerill FR. Tetracyclines, chloramphenicol, erythromycin, clindamycin and metronidazole. Mayo Clin Proc 1991; 66: $1270-80$.

11 Mauger TF. Antimicrobials. In: Mauger TF, Craig EL, eds. Havener's ocular pharmacology. 6th ed. St Louis: Mosby, 1994: 274-8.

12 Hanna C, Massey JY, Hendrickson RO, Williamson J, Jones EM, Wilson P. Ocular penetration of chloramphenicol. Arch Ophthalmol 1978; 96: 1258-61.

13 Lafarge-Frayssinet C, Robbana-Barnat S, Frayssinet C, Toucas L, Decloitre F. Cytotoxicity and DNA damaging potency of chloramphenicol and six metabolites: a new evaluation in human lymphocytes and Raji cells. Mutation Res 1994; 320: 207-15.

14 Aragones JV, Eriksen SP. Stable chloramphenicol solution for ocular infections. Am $\mathscr{f}$ Ophthalmol 1968; 66: 104-6.

15 Sinclair NM, Leigh DA. A comparison of fusidic acid viscous eye drops and chloramphenicol eye ointment in acute conjunctivitis. Therapeutic Res 1988; 44: 468-74.

16 Beasley H, Boltralik J, Baldwin HA. Chloramphenicol in aqueous humor after topical application. Arch Ophthalmol 1975; 93: 184-5.

17 Reynolds JEF, ed. Martindale: the extra pharmacopoeia. 30th ed. London: The Pharmaceutical Press, 1993.

18 Leeming JP, Diamond JP, Trigg R, White L, Hoh HD, Easty D. Ocular penetration of topical ciprofloxacin and norfloxacin drops and their effect upon eyelid flora. Brf Ophthalmol 1994; 78: 546-8.

19 Power WJ, Collum LM, Easty DL, Bloom PA, Laidlaw DA, Libert J, et al. Evaluation of efficacy and safety of ciprofloxacin ophthalmic solution versus chloramphenicol. Eur f Ophthalmol 1993; 3: 77-82.

20 Erslev AJ. Drug-induced blood dyscrasias: I. Aplastic anaemia. $\mathfrak{F} A M A$ 1964; 188: 159-60.

21 Wallerstein RO, Condit PK, Kasper CK, Brown JW, Morrison FR Statewide study of chloramphenicol therapy and fatal aplastic anaemia. Statewide study of chloramph

22 Trope GE, Lawrence JR, Hind VMD, Bunney J. Systemic absorption of topically applied chloramphenicol eyedrops. Br $\mathcal{f}$ Ophthalmol 1979; 63: 690-1.

23 Fraunfelder FT, Bagby GC, Kelly DJ. Fatal aplastic anaemia following topical administration of ophthalmic chloramphenicol. Am $\mathcal{f}$ Ophthalmol 1982; 93: 356-60.

24 Abrams SM, Degnan TJ, Vinciguerra V. Marrow aplasia following topical application of chloramphenicol eye ointment. Arch Intern Med 1980; 140: 576-7.

25 Nagao T, Mauer AM. Concordance for drug-induced aplastic anaemia in identical twins. N Engl ₹ Med 1969; 281: 7-11.

26 Faunfelder FT, Morgan RL, Yunis AA. Blood dyscrasias and topical ophthalmic chloramphenicol. Am ₹ Ophthalmol 1993; 115: 812-3.

27 Rosenthal RL, Blackman A. Bone-marrow hypoplasia following use of chloramphenicol eye drops. $\mathscr{F} A M A$ 1965; 191: 148-9.

28 Carpenter G. Chloramphenicol eye-drops and marrow aplasia. Lancet 1975 ii: $326-7$.

29 Dutro MP. Chloramphenicol and aplastic anaemia. Am $\mathcal{f}$ Ophthalmol 1981; 92: 870-1.

30 Stevens JD, Mission GP. Ophthalmic use of chloramphenicol [Letter]. Lancet 1987; ii: 1456.

31 Mandell GL, Sande MA. Penicillins, cephalosporins, and other beta-lactam antibiotics. In: Goodman Gilman A, Rall TW, Nies AS, Taylor P, ed. The pharmacological basis of therapeutics. Vol II. 8th ed. Singapore: McGrawpharmacological basis of therapeut

32 Gassner B, Wuethrich A. Pharmacokinetic and toxocological aspects of the medication of beef-type calves with an oral formulation of chloramphenicol palmitate. F Vet Pharmacol Ther 1994; 17: 279-83.

33 Page SW. Chloramphenicol. I: Hazards of use and current regulatory environment. Aust Vet f 1991; 68: 1-2. 